PROCEEDINGS OF THE

AMERICAN MATHEMATICAL SOCIETY

Volume 128, Number 10, Pages 3047-3056

S 0002-9939(00)05679-3

Article electronically published on March 3, 2000

\title{
A "UNIVERSAL" DYNAMICAL SYSTEM GENERATED BY A CONTINUOUS MAP OF THE INTERVAL
}

\author{
DAVID POKLUDA AND JAROSLAV SMÍTAL
}

(Communicated by Michael Handel)

\begin{abstract}
In this paper we show that there is a continuous map $f: I \rightarrow I$ of the interval such that any $\omega$-limit set $W$ of any continuous map $g: I \rightarrow I$ can be transformed by a homeomorphism $I \rightarrow I$ to an $\omega$-limit set $\tilde{W}$ of $f$. Consequently, any nowhere-dense compact set and any finite union of compact intervals is a homeomorphic copy of an $\omega$-limit set of $f$.
\end{abstract}

\section{INTRODUCTION}

Let $I=[0,1]$ be the unit interval in $R$ and let $\mathcal{C}(I, I)$ denote the class of continuous functions from $I$ to $I$. For $f \in \mathcal{C}(I, I), f^{0}$ is the identity function, and for any positive integer $n$, the $n$-th iterate $f^{n}$ is defined by $f^{n}=f \circ f^{n-1}$. A set $W \subset I$ is called an $\omega$-limit set for $f$ if there is an $x \in I$ such that $W$ is the cluster set of the sequence $\left\{f^{n}(x)\right\}$. Denote this set by $\omega_{f}(x)$, and let $\omega_{f}$ be the class of all $\omega$-limit sets of $f$. Any $W \in \omega_{f}$ is a non-empty closed subset of $I$ and $f(W)=W$.

The following characterization of $\omega$-limit sets is due to Agronsky et al. 11: a non-empty compact set $W \subset I$ is an $\omega$-limit set of a map $f \in \mathcal{C}(I, I)$ if and only if $W$ is either a finite collection of compact intervals or nowhere dense (by interval we mean a non-degenerate one, exceptions are stated explicitely). Recall that the necessary condition was proved already in the sixties by Sharkovsky [8]; a simpler proof of the sufficient condition is given in [5]. In fact, 5] contains a stronger result: any infinite nowhere dense compact set $W$ is an $\omega$-limit set of homoclinic type for a suitable continuous map of $I$. A characterization of sets in $\omega_{f}$, for any fixed continuous $f$, is given in [3]; it involves a transportation condition on the sets. The system $\omega_{f}$ equipped with the Hausdorff metric is a compact set [3].

In view of the above-mentioned facts there is the following natural problem: how large can the system $\omega_{f}$ be? In [7] Keller gives a simple example of a function $f: I \rightarrow I$ continuous everywhere except for a single point such that any nowhere dense compact set $F \subset I$ has a homeomorphic copy $\tilde{F}$ in $\omega_{f}$; the corresponding homeomorphism can be extended to the whole of $I$. By Evans et al. [6], if $f \in$ $\mathcal{C}(I, I)$ has periodic orbits of all periods, then any non-empty countable compact set has a homeomorphic copy in $\omega_{f}$. This homeomorphism, however, cannot be in general extended from $F$ to the whole interval $I$. In this paper we prove that there

Received by the editors November 1, 1998.

1991 Mathematics Subject Classification. Primary 58F12, 58F08, 58F03, 26 A18.

This research was supported, in part, by contract No. 201/97/0001 from the Grant Agency of the Czech Republic. Support of this institution is gratefully acknowledged. 
is a "universal" continuous function, up to homeomorphisms of the interval, solving a problem formulated by A. M. Bruckner (cf., e.g., [4]). Our main result is the following one.

Theorem 1.1. There is a map $f \in \mathcal{C}(I, I)$ such that, for any non-empty compact set $F \subset I$ which is either nowhere dense or is the union of finitely many nondegenerate intervals, there is a homeomorhism $\varphi$ of I such that $\varphi(F)$ is an $\omega$-limit set of $f$.

Clearly, the condition "up to homeomorphism" cannot be omitted, and the function involved cannot be simple. In our case $f$ is strongly irregular, having infinite variation on any open interval which intersects a Cantor set $C_{f}$. Moreover, the $\omega$-limit sets of $f$ which are contained in $C_{f}$ form our universal system for infinite nowhere dense $\omega$-limit sets. Instead of Theorem 1.1 it is more convenient to prove the following equivalent statement.

Theorem 1.2. There is a countable family of maps $f_{n} \in \mathcal{C}(I, I), n=1,2, \ldots$, such that, for any non-empty compact set $F \subset I$ which either is nowhere dense or is the union of finitely many non-degenerate intervals, there is a homeomorhism $\varphi$ of I such that $\varphi(F)$ is an $\omega$-limit set of $f_{n}$, for some $n$.

The equivalence follows by the "diagonalization" method. Assume that Theorem 1.2 is true, and let $\left\{I_{n}\right\}$ be pairwise disjoint compact intervals in $I$. Let $f \in \mathcal{C}(I, I)$ be any map such that $f \mid I_{n}$ is conjugated to $f_{n}$ (i.e., $f_{n} \circ \psi=\psi \circ\left(f \mid I_{n}\right)$, where $\psi$ is a homeomorphism $I_{n} \rightarrow I$ ). Then $f$ is the universal function from Theorem 1.1 The converse implication is obvious.

Remark 1.3. For any positive integer $k$, it is easy to find a continuous map which has an $\omega$-limit set consisting of exactly $k$ disjoint compact periodic intervals and hence, a $k$-cycle. So, to prove Theorem 1.2 it suffices to show that it is true for the infinite nowhere dense compact sets $F$.

In this paper we use standard terminology and notation (see, e.g., 2]). However, some notions should be fixed. A Cantor set is any nowhere dense non-empty compact set without isolated points. A homeomorphic copy of a set $A \subset I$ is a copy with respect to an order-preserving homeomorphism (which can be extended to the whole interval). The following notion of an $\omega$-limit set of homoclinic type plays a central role in our construction.

Definition 1.4. Let $W$ be a nowhere dense compact set, and $A=\left\{a_{0}, \ldots, a_{k-1}\right\} \neq$ $\emptyset$ a subset of the set of limit points of $W$. Assume there is a system $\left\{W_{n}^{i}\right\}_{n=1}^{\infty}$, $i=0, \ldots, k-1$, of non-empty pairwise disjoint compact subsets of $W$ such that $W \backslash \bigcup_{i, n} W_{n}^{i}=A$ and $\lim _{n \rightarrow \infty} W_{n}^{i}=a_{i}$, for any $i$. Let $f: W \rightarrow W$ be a continuous map and let $A$ be a $k$-cycle of $f$ such that, for any $i(\bmod k), f\left(a_{i}\right)=a_{i-1}$, $f\left(W_{n}^{i}\right)=W_{n-1}^{i-1}$ if $n>1$ and $f\left(W_{1}^{i}\right)=a_{i-1}$. Then $W$ is called a homoclinic set of order $k$ with respect to $f$.

A homoclinic set $W$ of $f$ is an $\omega$-limit set of $f$ if, for any $a \in A$ and any open set $U$ intersecting $W, f^{k}(U)$ is a neighborhood of $a$, for some $k$ (cf. Lemma 4.1 below).

The paper is organized as follows. Section 2 contains preliminaries on mappings between uncountable nowhere dense compact sets (Lemma 2.3). This and the 
Itinerary Lemma 4.1 are the tools allowing us to construct a universal function for uncountable nowhere dense compact sets (Proposition 5.1). The universal function even need not have the 3c-property (Definition 3.1 and Lemma 4.2), GC-maps (Definition [2.2) are quite sufficient in this case. However, the "countable part" of the construction is not so simple. Preliminaries are given in Section 3, with Propositions 3.6 and 3.7 as the main results (they play a role similar to Lemma 2.3 in the uncountable case). Section 5 contains the main constructions.

\section{Preliminaries - uncountable case}

Denote by $Q$ and $R$ the sets of rational and real numbers. For $F \subset R$, let $\bar{F}$ be the closure of $F$, and $F^{+}$or $F^{-}$the set of right- or left-isolated points in $F$, respectively. For $S \subset R$, let $\mathcal{E}(S)$ be the family of nowhere dense compact sets $P$ such that $P^{+} \cup P^{-} \subset S$. The following result must be known but we are not able to give a reference. Its proof uses standard techniques (cf., e.g., [9]).

Lemma 2.1. Let $F$ be a Cantor set in $\mathcal{E}(S)$. Then any nowhere dense compact set $A$ has a homeomorphic copy $\tilde{A} \subset F$ in $\mathcal{E}(S)$.

Proof. We may assume that $A$ is infinite, $\min A=\min F=\alpha$ and $\max A=$ $\max F=\beta$. Let $\mathcal{I}=\left\{I_{n}\right\}_{n=1}^{\infty}$ and $\mathcal{J}=\left\{J_{n}\right\}_{n=1}^{\infty}, J_{n}=\left(a_{n}, b_{n}\right)$, be an enumeration of the intervals complementary to $A$ or $F$, respectively. Assume that $I_{1}=J_{1}$ and $I_{2}=J_{2}$ are the intervals complementary to $[\alpha, \beta]$. Consider $\mathcal{I}$ and $\mathcal{J}$ as ordered sets with the natural ordering. To get $\tilde{A}$ it suffices to get a system $\mathcal{K}=\left\{K_{n}\right\}_{n=1}^{\infty}$, $K_{n}=\left(u_{n}, v_{n}\right)$, of intervals complementary to $\tilde{A}$ and use the fact that $A$ and $\tilde{A}$ are homeomorphic if and only if $\mathcal{I}$ and $\mathcal{K}$ are.

Let $K_{1}=I_{1}$ and $K_{2}=I_{2}$. Assume by induction that $K_{1}, \ldots, K_{n}$ are defined such that their end-points are end-points of intervals from $\mathcal{J}$ and $I_{i} \mapsto K_{i}$ is a homeomorphism between $\left\{I_{1}, \ldots, I_{n}\right\}=\mathcal{I}_{n}$ and $\left\{K_{1}, \ldots, K_{n}\right\}=\mathcal{K}_{n}$. Let $I_{j}$ be the left neighbor of $I_{n+1}$ in $\mathcal{I}_{n}$, and let $k$ be the smallest index such that $J_{k}$ is disjoint from $\bigcup \mathcal{K}_{n}$, and $K_{j}$ is the left neighbor of $J_{k}$ in $\mathcal{K}_{n}$. If there are intervals from $\mathcal{I}$ between $I_{j}$ and $I_{n+1}$, put $u_{n+1}=a_{k}$; otherwise let $u_{n+1}=v_{j}$. Similarly find $v_{n+1}$. The choice of $J_{k}$ at any step implies that $\tilde{A}$ contains no interval. Thus both $A$ and $\tilde{A}$ are nowhere dense and, consequently, $I_{i} \mapsto K_{i}$ is a homeomorphism between $\mathcal{I}$ and $\mathcal{K}$.

Definition 2.2. Let $X$ and $Y$ be compact intervals. Then $\tau \in \mathcal{C}(X, Y)$ is called a generalized Cantor map, or $G C$-map, provided there is a Cantor set $C=C_{\tau} \in \mathcal{E}(Q)$ contained in the interior of $X$ such that the family $\mathcal{S}_{\tau}$ of closures of the intervals complementary to $C$ in $X$ has the following properties.

(i) $\mathcal{S}_{\tau}$ is the family of the maximal intervals of constancy of $\tau$;

(ii) for any $x \in X, \tau(x) \in Q$ if and only if $x \in \bigcup S_{\tau}$.

Let $\tau \in \mathcal{C}(X, Y)$ be a GC-map. For any $L \in \mathcal{S}_{\tau}$, let $C_{L}$ be a Cantor set in $\mathcal{E}(Q)$ contained in the interior of $L$. Denote $D_{\tau}=C_{\tau} \cup \bigcup_{L \in \mathcal{S}_{\tau}} C_{L}$. Then $D_{\tau}$ is a Cantor set contained in the interior of $X$ and $D_{\tau} \in \mathcal{E}(Q)$. Let $\mathcal{R}_{\tau}$ be the family of closures of the intervals complementary to $D_{\tau}$. For any $L \in \mathcal{R}_{\tau}$ let $E_{L}$ be a Cantor set in $\mathcal{E}(Q)$ containing the end-points of $L$ which are interior to $X$. Denote $E_{\tau}=D_{\tau} \cup \bigcup_{L \in \mathcal{R}_{\tau}} E_{L}$. Again, $E_{\tau} \in \mathcal{E}(Q)$. 
Lemma 2.3. Let $X$ and $Y$ be compact intervals, $\tau \in \mathcal{C}(X, Y)$ a surjective $G C$ map, and $A \subset Y, B \subset X$ nowhere dense compact sets such that $B$ is uncountable and $A \in \mathcal{E}(Q)$. Then there is a homeomorphism $\psi$ of $X$ such that $\tilde{B}=\psi(B) \in$ $\mathcal{E}\left(E_{\tau}^{+} \cup E_{\tau}^{-}\right)$and $\tau(\tilde{B})=A$.

Proof. By Definition 2.2, $M=\tau^{-1}(A \cap Q)$ is the union of intervals from $\mathcal{S}_{\tau}$. Let $K$ be the set of points in $D_{\tau}$ interior to $M$. Then $C=\bar{K}$ is a Cantor set, and $\tau(C)=\overline{\tau(K)}=\overline{\tau(M)}=\overline{A \cap Q}=A$; the last equality follows since $A \in \mathcal{E}(Q)$ and $\overline{A^{+}}=A$.

Let $J=(a, b)$ be an interval complementary to $C$, with $\min C<a<b<\max C$. Then $a \in D_{\tau}^{+}$and $b \in D_{\tau}^{-}$. Indeed, $\tau(a)$ and $\tau(b)$ are points in $A$; if both are in $Q$ the statement is true by definition of $\tau$. On the other hand if, say, $\tau(a) \notin Q$, then any one-sided neighborhood of $\tau(a)$ contains a point in $A \cap Q$ since $A \in \mathcal{E}(Q)$. By Definition 2.2, $\tau$ cannot be constant on $J$; hence $\tau(J)$ contains a one-sided neighborhood of $\tau(a)$ and consequently $J$ intersects $M$ - a contradiction.

Let $B=B_{1} \cup B_{2}$, where $B_{1}$ is a Cantor set and $B_{2}$ is countable. Without loss of generality we may assume that the system $\mathcal{M}_{B}$ of the closures of the complementary intervals of $B_{1}$ with natural ordering has the first and the last element. Hence it is similar to the corresponding system $\mathcal{M}_{C}$ for $C$. Let $\psi: X \rightarrow X$ be a homeomorphism with $\psi\left(B_{1}\right)=C$. Then for any $L$ in $\mathcal{M}_{B}, \psi(L)$ is in $\mathcal{M}_{C}$, and $L \cap B$ is non-empty (since $L$ is closed), countable and compact. Apply Lemma 2.1 to get a homeomorphic copy $\tilde{B}_{L}$ of $B \cap L$ such that $\tilde{B}_{L} \in\left(E_{\tau}^{+} \cup E_{\tau}^{-}\right), \tilde{B}_{L} \subset \psi(L)$, $\min \tilde{B}_{L}=\min \psi(L)$, and $\max \tilde{B}_{L}=\max \psi(L)$. Finally, let $\tilde{B}$ be the union of $C$ and all $\tilde{B}_{L}$, for $L \in \mathcal{M}_{B}$.

\section{Preliminaries - Countable CASE}

We start with some terminology and notation. For $A, B \subset R$ write $A \leq B$ if $x \leq y$ whenever $x \in A$ and $y \in B$, and write $A<B$ if $A \leq B$ and $A \neq B$. Let $\mathcal{J}$ be a system of intervals. For any $J \in \mathcal{J}$, let $\mathcal{J}_{J}$ be a finite system of intervals (with disjoint interiors) which form a partition of $J$. Then $\bigcup_{J \in \mathcal{J}} \mathcal{J}_{J}$ is called a finite refinement of $\mathcal{J}$.

Let $f \in \mathcal{C}(X, Y)$, and $K \subset X, L \subset Y$ be compact intervals. Then $K f$-covers $L$ three times, if $K$ contains three non-overlapping intervals (i.e., intervals with disjoint interiors) $K_{1}, K_{2}, K_{3}$ such that $f\left(K_{i}\right) \supset L$, for any $i$. Moreover, $f \mid K$ is an $i$-map if there are points $u<v$ in $K$ such that $f(u)<f(v)$ are the end-points of $f(K) ; f \mid K$ is a $d$-map if there are points $u<v$ in $K$ such that $f(u)>f(v)$ are the end-points of $f(K)$. Note that one map may well be both an $i$-map and a $d$-map.

Definition 3.1. A map $f \in \mathcal{C}(X, Y)$ has the 3-coverings property, or 3c-property if the following three conditions are satisfied:

(a) $X f$-covers $Y$ three times;

(b) $f$ attains a rational value at any point of local extremum;

(c) if $K \subset X$ and $L \subset Y$ are compact intervals such that $f(K) \supset L$, then there are finite partitions $K_{1}<K_{2}<\cdots<K_{n}$ of $K$ and $L_{1}<L_{2}<\cdots<L_{n}$ of $L$ if $f \mid K$ is an $i$-map, and $L_{n}<L_{n-1}<\cdots<L_{1}$ otherwise, into non-overlapping intervals such that the dividing points in $L$ are rational numbers and $K_{i} f$-covers $L_{i}$ three times, for any $i$.

Note that by (a) and (b), $Y$ must have rational end-points. 
Lemma 3.2. Let $\tau \in \mathcal{C}(X, Y)$ be a $G C$-map with the 3c-property, and let $\tau(a) \notin Q$. Then any one-sided neighborhood of a is mapped onto a neighborhood of $\tau(a)$ in $Y$.

Proof. Denote $\tau(a)=b$ and assume that, e.g., $\tau(K)=L$, where $K=[a, a+\delta]$, $L=[b, b+\varepsilon]$, and $\delta, \varepsilon$ are positive. Then by Definition 3.1 (c) there is a compact interval $K^{\prime}$ contained in the interior of $K$ such that $b \in \tau\left(K^{\prime}\right)$. Thus, there is a $c$ interior to $K$ such that $\tau(c)=b$; hence $\tau$ has a local minimum at $c$, contrary to Definition 3.1(b).

A sequence $\left\{I_{n}\right\}_{n=0}^{\infty}$ of intervals is monotone if it is monotone with respect to the ordering $<$ among sets, i.e., if this sequence accumulates to the right or to the left.

Lemma 3.3. Let $\tau \in \mathcal{C}(X, Y)$ be a GC-map with the 3c-property. Let $\mathcal{I}=\left\{I_{n}\right\}_{n=1}^{\infty}$ and $\mathcal{J}=\left\{J_{n}\right\}_{n=1}^{\infty}$ be monotone sequences of compact subintervals of $X$ and $Y$, respectively such that the intervals in $\mathcal{I}$ are pairwise disjoint, and these in $\mathcal{J}$ are non-overlapping and have the end-points in $Q$. Finally, assume that there is a compact interval $K$ in $X$ such that $\tau(K)=Y, \tau \mid K$ is an i-map if the sequences $\mathcal{I}$ and $\mathcal{J}$ have the same orientation, and $\tau \mid K$ is a d-map otherwise.

Then there is a finite refinement $\tilde{\mathcal{J}}=\left\{\tilde{J}_{n}\right\}_{n=1}^{\infty}$ of $\mathcal{J}$, and a homeomorphism $\varphi: X \rightarrow X$ such that $\varphi\left(I_{n}\right) \subset K$ and $\varphi\left(I_{n}\right) \tau$-covers $\tilde{J}_{n}$ three times, for any $n$.

Proof. Assume first that both sequences are increasing and $K$ is a minimal interval such that $\tau(K)=Y$ and $\tau \mid K$ is an $i$-map. Let $u_{1}<v_{1}$ be the minimal points in $K$ such that $\tau\left(u_{1}\right)<\tau\left(v_{1}\right)$ are the end-points of $J_{1}$. By induction, let $u_{n+1}<v_{n+1}$ be points in $K$ such that $v_{n}<u_{n+1}$ and $\tau\left(u_{n+1}\right)<\tau\left(v_{n+1}\right)$ are the end-points of $J_{n+1}$. Moreover, since $\tau \mid K$ is an $i$-map, we can choose $v_{n+1}$ to be the minimal point satisfying the above conditions. Denote $K_{n}=\left[u_{n}, v_{n}\right]$. Then $\tau \mid K_{n}$ is an $i$-map, for any $n$. Consequently, since $\tau$ has the 3c-property, there are finite partitions $K_{n 1}<K_{n 2}<\cdots<K_{n k}$ of $K_{n}$, and $J_{n 1}<J_{n 2}<\cdots<J_{n k}$ of $J_{n}$ such that $K_{n i} \tau$ covers $J_{n i}$ three times. Rearrange all $J_{n i}$ into a monotone sequence $\tilde{\mathcal{J}}=\left\{\tilde{J}_{n}\right\}_{n=1}^{\infty}$. Denote by $\tilde{K}_{n i}$ a minimal compact subinterval of $K_{n i}$ which $\tau$-covers $J_{n i}$ three times. Since each $J_{n i}$ has rational end-points and $\tau$ is a GC-map, any neighbors $\tilde{K}_{n i}, \tilde{K}_{n j}$ are separated by an interval of constancy of $\tau$. Hence the intervals $\tilde{K}_{n i}$ can be rearranged into an increasing sequence $\tilde{\mathcal{I}}=\left\{\tilde{I}_{n}\right\}_{n=1}^{\infty}$ of pairwise disjoint compact intervals. So there is a homeomorphism $\varphi: X \rightarrow X$ such that $\varphi\left(I_{n}\right)=\tilde{I}_{n}$, for any $n$.

If both sequences are decreasing, the argument is similar. In the other case use the fact that $\tau \mid K$ is a $d$-map.

Let $A \subset R$ be a countable compact set, and let $\Omega$ be the first uncountable ordinal. Define a non-increasing transfinite sequence $\left\{A_{\alpha}\right\}_{\alpha \in \Omega}$ of sets as follows: $A_{0}=A, A_{\gamma}=\bigcap_{\alpha<\gamma} A_{\alpha}$ if $\gamma$ is a limit ordinal, and $A_{\gamma}$ is the derivative (i.e., the set of limit points) of $A_{\gamma-1}$ otherwise. By the Baire-Hausdorff theorem, there is an ordinal $\beta<\Omega$ such that $A_{\beta}$ is non-empty and finite (and hence, $A_{\beta+1}=\emptyset$ ); denote this $\beta$ by $T(A)$, and call it the depth of $A$. The set $A_{T(A)}$ will be referred to as the center of $A$. We say that a sequence $\left\{I_{n}\right\}_{n=1}^{\infty}$ of intervals converges to a point $a$, and write $\lim _{n \rightarrow \infty} I_{n}=a$, if any neighborhood of $a$ contains all but a finite number of $I_{n}$. For countable compact sets $A$ and $B$ in $R$ put $A \prec B$ if either $T(A)<T(B)$, or $T(A)=T(B)$ and $\# A_{T(A)} \leq 2 \# B_{T(B)}$. 
Lemma 3.4. Let $A \subset R$ be a non-empty countable compact set with $T(A)=\alpha$, and $A_{\alpha}=\{a\}$. Then there is a sequence $\left\{J_{n}\right\}_{n=1}^{\infty}$ of pairwise disjoint compact intervals covering $A \backslash\{a\}$, and converging to $a$, which has one of the following forms:

$$
\begin{gathered}
J_{1}<J_{3}<\cdots<J_{2 n-1}<\cdots<a<\cdots<J_{2 n}<\cdots<J_{4}<J_{2}, \\
J_{1} \leq a<\cdots<J_{k}<\cdots<J_{3}<J_{2}, \\
J_{2}<J_{3}<\cdots<J_{k}<\cdots<a \leq J_{1},
\end{gathered}
$$

possibly with $J_{1}=\{a\}$ in cases (3.2) or (3.3), such that $J_{n} \cap A \prec J_{n+1} \cap A$ for each $n$. Moreover if $\left\{B_{n}\right\}$ are countable compact sets such that $B_{n} \prec A$, then intervals $\left\{J_{n}\right\}$ can be chosen such that $B_{n} \prec J_{n} \cap A$, for $n>1$.

Proof. Let $u=\min A, v=\max A, A^{l}=A \cap[u, a]$, and $A^{r}=A \cap[a, v]$. If $T\left(A^{r}\right)=\beta<\alpha$ put $J_{1}=[a, v]$. In this case, $T\left(A^{l}\right)=\alpha$. For $x \in[u, a), \psi(x)=$ $T(A \cap[u, x])<\alpha$ is a nondecreasing function, and $\lim _{x \rightarrow a} \psi(x)$ is $\alpha$ if $\alpha$ is a limit ordinal, and is $\alpha-1$ otherwise. Since $A$ is nowhere dense, there are compact pairwise disjoint intervals $J_{i}$, for $i>1$, satisfying (3.3) such that $\beta<T\left(A \cap J_{i}\right)<T\left(A \cap J_{i+1}\right)$ if $\alpha$ is limit, and $\beta \leq T\left(A \cap J_{i}\right)=\alpha-1$ otherwise. The case $T\left(A^{l}\right)<\alpha$ similarly leads to (3.2), and $T\left(A^{l}\right)=T\left(A^{r}\right)=\alpha$ to (3.1).

Lemma 3.5. Let $A$ be a countable compact set whose center is a singleton $\{a\}$. Then there are non-overlapping, possibly degenerate intervals $\left\{H_{n}\right\}_{n=1}^{\infty}$ with endpoints in $Q$, covering $A \backslash\{a\}$ such that $H_{1} \leq H_{3} \leq \cdots \leq a \leq \cdots \leq H_{4} \leq H_{2}$, $\lim _{n \rightarrow \infty} H_{n}=a$, and $H_{i} \prec A$, for $i \geq 1$.

Proof. Apply Lemma 3.4 to get intervals $\left\{J_{n}\right\}$. In case (3.2) find disjoint intervals $H_{2 n}$ such that $H_{2 n} \supset J_{n+1}$, for $n>0$. If $a$ is left-isolated, take $H_{1} \subset J_{1} \backslash\{a\}$, $H_{1} \supset J_{1} \cap A \backslash\{a\}$, and $H_{2 n-1}=\{a\}$, for $n>1$. Otherwise cover $J_{1} \cap A \backslash\{a\}$ by an increasing sequence $\left\{H_{2 n-1}\right\}$ of disjoint intervals. Similarly in the other cases.

Proposition 3.6. Let $X$ and $Y$ be compact intervals with rational end-points, and $\tau \in \mathcal{C}(X, Y)$ a $G C$-map with the 3c-property. Let $A \subset Y$ and $B \subset X$ be countable compact sets such that $A \in \mathcal{E}(Q)$ and $A \prec B$. Then there is a homeomorphism $\psi$ of $X$ such that $\psi(B) \in \mathcal{E}\left(D_{\tau}^{+} \cup D_{\tau}^{-}\right)$and $\tau(\psi(B))=A$.

Proof. The statement is obviously true if $T(A)=0$, so assume by transfinite induction that it is true whenever $T(A)<\alpha$, and let $T(A)=\alpha$.

Case 1. Assume that the center of $A$ is a singleton $A_{\alpha}=\{a\}$. Apply Lemma 3.5 to get intervals $H_{n}$. Since $A \prec B$ there are two disjoint compact intervals $U<V$ such that $T(B \cap U)=T(B \cap V)=\beta \geq \alpha,(B \cap U)_{\beta}=\{u\}$, and $(B \cap V)_{\beta}=\{v\}$, where $u$ and $v$ are end-points of $U$ and $V$, respectively. Apply Lemma 3.4 to $B \cap U$ to get a monotone sequence $\mathcal{I}_{u}=\left\{I_{n}^{u}\right\}$ of compact subintervals of $U$ which covers $B \cap U \backslash\{u\}$, such that $\lim _{n \rightarrow \infty} I_{n}^{u}=u$, and $B \cap I_{n}^{u} \succ A \cap H_{i}$, for $i \leq 2 n$. Similarly find $\mathcal{I}_{v}=\left\{I_{n}^{v}\right\}$ for $B \cap V$ such that $\lim _{n \rightarrow \infty} I_{n}^{v}=v$ and $B \cap I_{n}^{v} \succ A \cap H_{i}$, for $i \leq 2 n$.

If both sequences $\mathcal{I}_{u}$ and $\mathcal{I}_{v}$ are increasing, let $K_{0}, K_{1}$ be non-overlapping compact intervals with $\tau\left(K_{0}\right)=\tau\left(K_{1}\right)=Y$ such that $\tau \mid K_{0}$ is an $i$-map and $\tau \mid K_{1}$ a $d$-map. Such intervals exist since $\tau$ is a 3c-map. If $K_{0}<K_{1}$ apply twice Lemma 3.3, first to $K=K_{0}, \mathcal{I}=\mathcal{I}_{u}$ and $\mathcal{J}=\left\{H_{2 n-1}\right\}$, and then to $K=K_{1}, \mathcal{I}=\mathcal{I}_{v}$ and $\mathcal{J}=\left\{H_{2 n}\right\}$, to get a homeomorphism $\varphi$ of $I$ such that the intervals from $\tilde{\mathcal{I}}_{u}=\varphi\left(\mathcal{I}_{u}\right)$ are contained in $K_{0}$, and these from $\tilde{\mathcal{I}}_{v}=\varphi\left(\mathcal{I}_{v}\right)$ in $K_{1}$. For any interval 
$L$ in $\tilde{\mathcal{I}}_{u} \cup \tilde{\mathcal{I}}_{v}, \tau \mid L$ is a GC-map in $\mathcal{C}(L, \tau(L))$ with the 3c-property. Since $A \cap \tau(L)$ and $B \cap L$ satisfy the hypothesis of the proposition, and the depth of $A \cap \tau(L)$ is less than $\alpha$, by the induction hypothesis there is a homeomorphism $\varphi_{L}$ of $L$ such that $\varphi_{L}(B \cap L)$ is mapped by $\tau$ onto $A \cap \tau(L)$. It remains to insert a homeomorphic copy of $B \backslash(U \cup V)$ into $\mathcal{E}\left(D_{\tau}^{+} \cup D_{\tau}^{-}\right) \backslash \varphi(U \cup V)$ such that it is mapped into $A$. But this is easy since $\tau$ has 3c-property and the sets $\varphi(U)$ and $\varphi(V)$ are separated by intervals of constancy of $\tau$ - cf. Definition 2.2 and Lemma 2.1.

If $K_{0}>K_{1}$, exchange above $\mathcal{I}_{u}$ and $\mathcal{I}_{v}$. The argument is similar, when $\mathcal{I}_{u}$ and $\mathcal{I}_{v}$ are decreasing: it suffices to exchange above $\left\{H_{2 n-1}\right\}$ and $\left\{H_{2 n}\right\}$. If the sequences have opposite orientation, choose intervals $K_{0}$ and $K_{1}$ such that $\tau \mid K_{0}$ and $\tau \mid K_{1}$ are both $i$-maps or both $d$-maps.

Case 2. Let $\# A_{\alpha}=m>1$, where $\alpha=T(A)$. Then there are disjoint compact intervals $I_{1}<I_{2}<\cdots<I_{m}$ covering $A$ such that the depth of any $A_{i}=A \cap I_{i}$ is $\alpha$, and its center is a singleton. Find a similar decomposition $B_{1}<B_{2}<\cdots<B_{m}$ of $B$ such that any pair $A_{i}, B_{i}$ meets the hypothesis of Proposition [3.6 and apply the result proved in Case 1.

Proposition 3.6 and the next result make it possible to transform any infinite countable compact set $A$, by a suitable homeomorphism of the interval, to a homoclinic set of $\tau$.

Proposition 3.7. Let $W \subset R$ be a countably infinite compact set, with depth $T(W)=\alpha$ and center $W_{\alpha}=A=\left\{a_{0}, \ldots, a_{k-1}\right\}$. Then there is a system $\left\{W_{n}^{i}\right\}_{n=1}^{\infty}$, $i=0, \ldots, k-1$, of non-empty pairwise disjoint compact portions of $W$ with the following properties:

(i) $W \backslash \bigcup_{i, n} W_{n}^{i} \subset A$;

(ii) $\lim _{n \rightarrow \infty} W_{n}^{i}=a_{i}$, for any $i$;

(iii) $W_{n-1}^{i-1} \prec W_{n}^{i}$, for any $n>1$, and any $i$ taken $\bmod k$.

Proof. Find disjoint compact intervals $I_{0}, \ldots, I_{k-1}$ covering $W$ such that $a_{i} \in I_{i}$. Apply Lemma 3.4 (with all $B_{n}$ empty) to any $W^{i}=I_{i} \cap W$ to get intervals $\left\{J_{n}^{i}\right\}$. A suitable decomposition of $W$ can be constructed by amalgamating the portions $W \cap J_{n}^{i}$ into bigger pieces. Let $W_{1}^{i}=J_{1}^{i} \cap W$. Let $\beta_{i}=T\left(W_{1}^{i}\right)$; then $\beta=\max _{i} \beta_{i}<\alpha$. Note that $W^{i} \backslash W_{1}^{i}$ is a portion of $W^{i}$.

Let $s$ be the minimal index such that $J_{s}^{i}$ is disjoint from $W_{1}^{i}$. Let $J_{s_{1}}^{i}=$ $J_{s}^{i}, J_{s_{2}}^{i}, \ldots, J_{s_{p}}^{i}$ be a minimal portion of intervals (i.e., the system of intervals $J_{j}^{i}$ contained in an interval) from one side of $a_{i}$ and disjoint from $W_{1}^{i}$ such that $\left(J_{s_{1}}^{i} \cup J_{s_{2}}^{i} \cup \cdots \cup J_{s_{p}}^{i}\right) \cap W \succ W_{1}^{i-1}$. Such a finite portion exists by Lemma 3.4. Put $W_{2}^{i}=\left(J_{s_{1}}^{i} \cup J_{s_{2}}^{i} \cup \cdots \cup J_{s_{p}}^{i}\right) \cap W$. The sets $W_{n}^{i}$ are defined similarly by induction.

\section{General Results}

An elementary result, which is well-known as the Itinerary Lemma, says that for any $f \in \mathcal{C}(I, I)$ and any sequence $\left\{U_{n}\right\}_{n=0}^{\infty}$ of compact intervals such that $f\left(U_{n}\right) \supset U_{n+1}$, for any $n$, there is a point $x$ such that $f^{n}(x) \in U_{n}$, for any $n$. The following result is its generalization.

Lemma 4.1. Let $f \in \mathcal{C}(I, I)$, and let $P$ be a countable set, $\left\{U_{n}\right\}_{n=0}^{\infty}$ a sequence of compact intervals such that $f\left(U_{n}\right) \supset U_{n+1}$ and $U_{n} \cap P \neq \emptyset$, for any $n$. Let any $p \in P$ be an element of $U_{n}$, for infinitely many $n$, and $\lim _{n \rightarrow \infty}$ diam $U_{n}=0$. Then there is an $x \in U_{0}$ such that $\omega_{f}(x)=\bar{P}$. 
Proof. By the Itinerary Lemma, there is an $x \in U_{0}$ such that $f^{n}(x) \in U_{n}$, for any $n$. Since $\omega_{f}(x)$ is closed, to prove the lemma it suffices to show that $P \subset \omega_{f}(x) \subset \bar{P}$. The first inclusion is trivial since, for any $p \in P$, both $p$ and $f^{n}(x)$ belong to $U_{n}$, for infinitely many $n$. To prove the other one, let $a \in \omega_{f}(x)$, and let $U_{\varepsilon}$ be the open $\varepsilon$-neighborhood of $a$. Then there is a subsequence $\left\{n_{k}\right\}$ such that $f^{n_{k}}(x) \in U_{\varepsilon}$, for any $k$, and hence, $U_{n_{k}} \subset U_{2 \varepsilon}$, for any sufficiently large $k$. Thus, $U_{2 \varepsilon} \cap P \neq \emptyset$ and consequently, $a \in \bar{P}$.

Lemma 4.2. Let $X$ and $Y$ be compact intervals, $Y$ with rational end-points, and let $C \subset \mathcal{E}(Q)$ be a Cantor set contained in the interior of $X$. Then there is a $G C$-map $\tau \in \mathcal{C}(X, Y)$ with the 3c-property such that $C_{\tau}=C$.

Proof. Let $\mathcal{S}=\left\{I_{n}\right\}_{n=1}^{\infty}$ be the system of closures of intervals complementary to $C$ in $X$, and let $\left\{r_{n}\right\}_{n=0}^{\infty}$ be an enumeration of $Q \cap Y$. Assume without loss of generality that $r_{0}=\min Y, r_{1}=\max Y, I_{1}<I_{j}<I_{2}$ for $j>2$, and $I_{1}<I_{3}<I_{4}<I_{2}$. Define a sequence $\left\{\tau_{n}\right\}_{n=1}^{\infty}$ of maps in $\mathcal{C}(X, Y)$ as follows. Let $\tau_{1}(x)=r_{0}$ for $x \in I_{1} \cup I_{4}$, $\tau_{1}(x)=r_{1}$ for $x \in I_{3} \cup I_{2}$, and let $\tau_{1}$ be linear on the intermediate intervals. Next assume by induction that $\tau_{n}$ is constant on any interval from a finite system $\mathcal{S}_{n} \subset \mathcal{S}$, and is linear and non-constant on any intermediate interval. Moreover, $\tau_{n}$ takes on any value $r_{0}, r_{1}, \ldots, r_{n}$, but only on the intervals of constancy. Hence, $T=\tau_{n}^{-1}\left(r_{n+1}\right)$ is a finite set. For any $t \in T$ find neighbor intervals $U_{t}<V_{t}$ in $\mathcal{S}_{n}$ such that $t$ is between them. Let $I_{k(1)}, \ldots, I_{k(5)}$ be the first five intervals from $\mathcal{S} \backslash \mathcal{S}_{n}$ such that $U_{t}<I_{k(1)}<\cdots<I_{k(5)}<V_{t}$. Let $\tau_{n+1}(x)=\tau_{n}(x)$ for $x \in \bigcup \mathcal{S}_{n}, \tau_{n+1}\left|I_{k(2)}=\tau_{n}\right| V_{t}, \tau_{n+1}\left|I_{k(4)}=\tau_{n}\right| U_{t}$, and $\tau_{n+1} \mid I_{k(1)} \cup I_{k(3)} \cup I_{k(5)}=$ $r_{n+1}$, and let $\tau_{n+1}$ be linear on the intermediate intervals. Thus, $\mathcal{S}_{n} \subset \mathcal{S}_{n+1}$, and $\left\|\tau_{n+1}-\tau_{n}\right\| \leq \delta_{n}$, where $\delta_{n}$ is the length of maximal interval disjoint from $\left\{r_{1}, \ldots, r_{n}\right\}$. Consequently, $\tau=\lim _{n \rightarrow \infty} \tau_{n}$ has the required properties.

\section{Proof of the MaIN THEOREM}

Proposition 5.1. Let $I=[0,1]$. Then there is a map $f \in \mathcal{C}(I, I)$ such that for any uncountable nowhere dense compact set $W \subset(0,1)$ there is a homeomorphism $\varphi$ of I such that $\varphi(W)$ is an $\omega$-limit set of $f$ of homoclinic type.

Proof. Let $a \in Q \cap(0,1)$, and let $\left\{J_{n}\right\}_{n=1}^{\infty}$ be a sequence of pairwise disjoint compact intervals with rational end-points in $(0,1)$ satisfying (3.1) such that $\lim _{n \rightarrow \infty} J_{n}=a$. Put $J_{0}=I$ and, for $n>0$, let $\tau_{n} \in \mathcal{C}\left(J_{n}, J_{n-1}\right)$ be a GC-map with the 3cproperty (cf. Lemma 4.2). For any $x \in J_{n}$, let $x_{D} \in D_{\tau_{n}}$ be a point such that $\operatorname{dist}\left(x, D_{\tau_{n}}\right)=\left|x-x_{D}\right|$. Define $f: I \rightarrow I$ as follows.

$$
f(x)= \begin{cases}\tau_{n}(x), & \text { if } x \in D_{\tau_{n}} \text { and } n>1, \\ a, & \text { if } x \in D_{\tau_{1}} \text { or } x=a, \\ \min \left\{1, \tau_{n}\left(x_{D}\right)+\left|x-x_{D}\right| \sin \left(1 /\left|x-x_{D}\right|\right)\right\}, & \text { if } x \in J_{n} \text { and } n \geq 1,\end{cases}
$$

and let $f$ be linear on the intermediate intervals. Clearly, $f$ is continuous.

Without loss of generality assume that $a \in W,\left\{J_{n}\right\}_{n=1}^{\infty}$ covers $W \backslash\{a\}$ and, for any $n, W_{n}=W \cap J_{n}$ is an uncountable compact set. By Lemma 2.1 find a set $\tilde{W}_{1} \in \mathcal{E}\left(D_{\tau_{1}}^{+} \cup D_{\tau_{1}}^{-}\right)$homeomorpic to $W_{1}$. Then, by Lemma 2.3 there is a homeomorphic copy $\tilde{W}_{2} \in \mathcal{E}\left(E_{\tau_{2}}^{+} \cup E_{\tau_{2}}^{-}\right)$such that $f\left(\tilde{W}_{2}\right)=\tau_{2}\left(\tilde{W}_{2}\right)=\tilde{W}_{1}$. By induction, let $\tilde{W_{n+1}} \in \mathcal{E}\left(E_{\tau_{n+1}}^{+} \cup E_{\tau_{n+1}}^{-}\right)$be homeomorphic to $W_{n+1}$ such that $f\left(\tilde{W_{n+1}}\right)=\tilde{W}_{n}$. Denote $\tilde{W}=\{a\} \cup \bigcup_{n=1}^{\infty} \tilde{W}_{n}$. Then $\tilde{W}$ is homeomorphic to $W$. 
To finish the proof put $E=\bigcup_{n=1}^{\infty} E_{\tau_{n}} \cup\{a\}, P=\tilde{W} \cap\left(E^{+} \cup E^{-}\right)$, and let $\left\{p_{n}\right\}_{n=1}^{\infty}$ be a sequence of points in $P$ containing any point infinitely many times. Denote by $U_{n}^{\prime}$ the open interval with center $p_{n}$ and diameter $1 / n$ and note that any $U_{n}^{\prime}$ is eventually mapped by $f$ onto a neighborhood of $a$. Then apply Lemma 4.1 .

Proposition 5.2. Let $f$ be as in Proposition 5.1 and $W \subset(0,1)$ a countably infinite compact set, whose center is a singleton $\{a\}$. Let $\left\{W_{n}\right\}_{n=0}^{\infty}$ be disjoint compact portions of $W$ given by Proposition 3.7 and, for any $n$, let $J_{n}$ be the convex hull of $W_{n}$. If the intervals $J_{n}$ satisfy (3.1), then there is a homeomorphism $\varphi$ of I such that $\varphi(W)$ is an $\omega$-limit set of $f$ of homoclinic type.

Proof. The argument is almost the same as for Proposition 5.1. The main difference is that to get the sets $\tilde{W}_{n}$, for $n>1$, we apply Proposition [3.6] instead of Lemma 2.3.

Proposition 5.3. There are maps $g, h \in \mathcal{C}(I, I)$ with the following property: let $W \subset(0,1)$ be a countably infinite compact set, whose center is a singleton $\{a\}$. Let $\left\{W_{n}\right\}_{n=0}^{\infty}$ be disjoint compact portions of $W$ given by Proposition 3.7 and, for any $n$, let $J_{n}$ be the convex hull of $W_{n}$. If the intervals $J_{n}$ satisfy (3.2) or (3.3), then there is a homeomorphism $\varphi$ of I such that $\varphi(W)$ is an $\omega$-limit set of homoclinic type of $g$ or $h$, respectively.

Proof. In the proof of Proposition 5.1, modify the construction of $f$ so that instead of (3.1) assume (3.2) or (3.3), and instead of Lemma 2.3 apply Proposition 3.6.

Proposition 5.4. For any integer $k>0$ there is a countable family $\mathcal{M}_{k} \subset \mathcal{C}(I, I)$ such that any countably infinite compact set $W \subset I$ whose center has $k$ elements is mapped by a homeomorphism of I onto an w-limit set of homoclinic type of a map in $\mathcal{M}_{k}$.

Proof. The statement is true for $k=1$ by Propositions 5.2 and 5.3 For $k>0$ the argument is similar. Note that there is a countable family of all possible orderings (up to homeomorphisms) of portions $W_{n}^{i}$ given by Proposition 3.7 To any such ordering $\rho$ assign a map $f_{\rho} \in \mathcal{C}(I, I)$ similarly, as in Propositions 5.2 and 5.3 .

Proof of Theorem 1.2, It follows by Propositions [5.1, 5.4, and Remark 1.3.

\section{REFERENCES}

1. S. J. Agronsky, A. M. Bruckner, J. G. Ceder and T. L. Pearson, The structure of $\omega$-limit sets for continuous functions, Real Anal. Exchange 15 (1989/90), 483 - 510. MR 91i:26008

2. L. Block and W. Coppel, Dynamics in one dimension, Lecture Notes in Mathematics 1513 (1991), Springer, New York, Heidelberg, Berlin. MR 93g:58091

3. A. Blokh, A. M. Bruckner, P. D. Humke and J. Smítal, The space of $\omega$-limit sets of a continuous map of the interval, Trans. Amer. Math. Soc. 348 (1996), 1357 - 1372. MR 96j:58089

4. A. M. Bruckner, Some problems concerning the iteration of real functions, Atti Sem. Mat. Fiz. Univ. Modena 41 (1993), 195 - 203. MR 94h:58114]

5. A. M. Bruckner and J. Smítal, The structure of $\omega$-limit sets for continuous maps of the interval, Math. Bohemica 117 (1992), 42 - 47. MR 93a:26002

6. M. J. Evans, P. D. Humke, C. M. Lee, and R. O'Malley, Characterizations of turbulent onedimensional mappings via $\omega$-limit sets, Trans. Amer. Math. Soc. 326 (1991), 261 - 280; correction, ibid. 333 (1992), 939 - 940. MR 91j:58133 
7. P. S. Keller, Chaotic behavior of Newton's method, Real Anal. Exchange 18 (1992/93), 490 - 507. MR 94j:26008

8. A. N. Sharkovsky, Attracting and attracted sets, Soviet Math. Dokl. 6 (1965), $268-270$.

9. W. Sierpiński, Cardinal and Ordinal Numbers, Polish Scientific Publishers, Warsaw, 1958. MR 20:2288

Institute of Mathematics, Silesian University, 74601 Opava, Czech Republic

E-mail address: David.Pokluda@fpf.slu.cz

Institute of Mathematics, Silesian University, 74601 Opava, Czech Republic

E-mail address: smital@fpf.slu.cz 